\title{
Electromagnetic Field Analysis of Operation Space of the Switch in the Substation Based on the Time Domain Integration Method
}

\author{
ZHANG Jian-gong ${ }^{1}$, Zhao Jun $^{1}$, GAN zheyuan ${ }^{1}, Z H A N G$ yemao ${ }^{1}$ and $L U$ ying $^{2}$ \\ ${ }^{1}$ China Electric Power Research Institute , Wuhan, China \\ ${ }^{2}$ North China Electric Power University, Beijing 102206, China
}

\begin{abstract}
In this paper, the approximate calculation formula is used to calculate the horizontal electric field from lightning which has arbitrary current, with the help of time domain integral equation method solving the lightning induced over voltages on overhead lines. In this paper, the time domain integral equations of horizontal electric field is established. Taking the conductor axis current as the variable, the pulse function is used as the basis function, and the discretization equation is used in space and time. Using time stepping algorithm to solve the resulting linear equations. The calculation process of this method is simple, and it is proved that the method is consistent with the conclusion of the relevant literature.
\end{abstract}

\section{1 introduction}

Air-insulated substations (AIS) are the most common type of substation. Switching operation in the station will generate a strong spatial electromagnetic field. With the promotion of smart substations, the spatial electromagnetic filed generated by the switching operation may have an impact on the smart components placed in the field. Therefore, the calculation of the space electromagnetic field generated by the switching operation in the substation is the basis for analysing whether the intelligent component can operate safely and reliably. The research of this problem has been reported in many related literatures [1-2].

From the calculation method, the time domain method has many characteristics superior to the frequency domain method in calculating the transient electromagnetic field generated by the switching operation $^{[1]}$. In particular, there is no need to perform mutual transformation between the time domain and the frequency domain, and the physical quantity changes with time can be clearly expressed. Typical time domain calculation methods are the time domain finite difference (FDTD) method[2] and the multi-conductor transmission line method(MTL)[3]. Both methods are applied in the calculation of space electromagnetic fields in substations[4-6]. However, there is a are error in using this method to calculation the bending and vertical models, and these two line models are common in substations. Therefore, the Time-Domain Integration Equation (TDIE) method for analyzing the electromagnetic scattering of metal structures and surface structures of more complex structures is more suitable for the space electromagnetic field generated by switching operations in substations. However, there is a general problem of late instability in the process of applying time step for, calculation. This paper[7] proposes the method of using average elimination in both time domain and spatial domain; the literature[8] adopts a more complicated form. The wavelet-timeintegral equation method has discrete space-time basis functions and has better stability. These methods can be used for time-domain integral equation method to perform spatial electromagnetic field analysis of switching operations in substations.

In the paper, for the spatial electromagnetic field analysis of switch operation in substation, firstly, the approximate calculation formula is used to derive the calculation formula of the space electric field, then time domain integral equation of the metal structure of the line conductor model is established, and in order to improve the numerical stability, the time domain integral equation is applied. The method of solving this integral equation is engineering application examples are given.

\section{Calculation method}

\section{1 time domain calculation of space electric field}

In order to establish a time-domain integral equation based on the electric field boundary condition of the conductor surface, it is first necessary to derive the time domain calculation formula of the spatial electric field. Based on the calculation formula of electric field strength and dynamic position, the calculation method of the tangential component of the electric field strength on the surface of the line conductor can be obtained, as shown in equation( 1 ). 


$$
E_{l}(r, t)=\frac{\partial A_{l}(r, t)}{\partial t}+\partial \varphi(r, t) / \partial l
$$

Among them:

$E_{l}(r, t)$ is the tangential component of the electric field strength at the time $t$ of the wire segment at 1 at the $r$ position; ${ }^{A}(r, t)$ : Indicated along the wire ${ }^{l}$ Dynamic vector of surface current; ${ }^{\varphi(r, t)}$ Indicated along the wire

$l$ Surface axis current dynamic scalar.

In an infinite vacuum, the relationship between $A_{l}(r, t)$ and the surface current is :

$$
A_{l}(r, t)=\frac{\mu}{4 \pi} \int_{l^{\prime}} \frac{i\left(r, r^{\prime}, t-R / c\right)}{R} d l^{\prime}
$$

the relationship between $\varphi(r, t)$ and the surface charge is :

$$
\varphi(r, t)=\frac{1}{4 \pi \varepsilon} \int_{l^{\prime}} \frac{q\left(l, l^{\prime}, t-R / c\right)}{R} d l^{\prime}
$$

Among them:

$\varepsilon_{\text {is the dielectric constant }}$

$\boldsymbol{R}=|\boldsymbol{r}-\boldsymbol{r}|$ indicates the distance from the field point to the source point.

$I\left(l, l^{\prime}, t-R / c\right)$ and $q\left(l, l^{\prime}, t-R / c\right)$ separately is located

$r$ 'line current and line charge at the conductor segment in the ${ }^{t=R / c}$

According to the law of conservation of charge:

$i\left(r, r^{\prime}, t-R / c\right)=-\partial q\left(r, r^{\prime}, t-R / c\right) / \partial t$

Equation(3)can be re-displayed:

$$
\varphi(r, t)=\int_{t}\left(-\frac{1}{4 \pi \varepsilon} \int_{l^{\prime}} \frac{i\left(r, r^{\prime}, t-R / c\right)}{R} d l^{\prime}\right) d t
$$

To simplify the representation,

$$
\psi(r, t)=-\frac{1}{4 \pi \varepsilon} \int_{l^{\prime}} \frac{i\left(r, r^{\prime}, t-R / c\right)}{R} d l^{\prime}
$$

$\psi(r, t)=\partial \varphi(r, t) / \partial t$

By substituting equation(5)and (2) into equation(1), a time domain calculation form of the spatial electric field can be obtained.

\subsection{Establishment of time domain electric field integral equation}

The incident field and the scattered field on the surface of the thin-line structure conductor have the tangential component of the electric field strength equal[4], namely:

$$
\boldsymbol{l} \times\left(\boldsymbol{E}^{i}+\boldsymbol{E}^{s}\right)=0
$$

In order to solve the equations of the time domain integral equation method, discrete solution are needed in the spatial and temporal domains respectively.

When the airspace is discrete, the conductor axis is divided into $\mathrm{N}$ segments, an $\mathrm{d}$ the current is selected as the basis function of the piecewise uniform function, which is denoted as $i k, k=1,2 \ldots \mathrm{N}+1$. The integral along the wire acts as the inner product of the weight function. Therefore, when only spatial dispersion is considered, equation(1) is transformed into:

$$
\frac{\partial \Phi(r, t)}{\partial t}+\varphi\left(l_{k}, t\right)-\varphi\left(l_{k-1}, t\right)=0
$$

Among them,

$\Phi(r, t)$ is scalar potential, $A_{l}(r, t)$ is the result after the weighted integral operation; the scond term and the third term are integral results of the potential derivative along the direction of the wire segment 1 .which is:

$$
\int_{l_{k}} \frac{\partial \varphi(r, t)}{\partial l} d l=\varphi\left(l_{k}, t\right)-\varphi\left(l_{k-1}, t\right)
$$

When the time domain is discrete, the time stepping method is applied, and the weight function is the $\omega_{j}(t)=\delta\left(t-t_{j}\right)$ angle $\mathrm{j}$ is the number of time steps calculated.

Equation(8)can form the following differential relationship:

$$
\frac{\Phi_{k, j}-\Phi_{k, j-1}}{\Delta t}+\varphi_{k, j}-\varphi_{k-1, j}=0
$$

Equation(6)can form the following differential relationship:

$$
\varphi_{\mathrm{k}, j}=\varphi_{\mathrm{k}, j-1}+\psi_{\mathrm{k}, j} \bullet \Delta t
$$

Substituting equation (11) into equation(10):

$$
\frac{\Phi_{k, j}-\Phi_{k, j-1}}{\Delta t}+\varphi_{\mathrm{k}, j-1}+\psi_{\mathrm{k}, j} \cdot \Delta t-\varphi_{k-1, j}=0
$$

Combining equation(2)and (6)to get the following:

$$
\begin{gathered}
\Phi_{\mathrm{k}, j}=\sum_{i=1}^{N} \frac{\mu}{4 \pi} \iint_{l_{k}} \frac{i_{i, j-n}}{\left|r_{i}-r_{i}\right|} d l^{\prime} d l \\
\psi_{k, j}=-\sum_{i=1}^{N} \frac{1}{4 \pi \varepsilon} \int_{l_{i}} \frac{i_{i, j-n}}{\left|r_{k}-r_{i}\right|} d l^{\prime}
\end{gathered}
$$

Among them:

$$
j-n \triangleq(j-n) \bullet \Delta t \approx t_{j}-\left|r_{k}-r_{i}\right| / c \text {. }
$$

$n$ is a value indicating a time step difference;

$n_{\max }$ describe the ratio of distance between the conductor segment k away from the conductor segment and the k segment with $\mathrm{c}$

$n_{\min }=0$ is the distance between the axis of section $\mathrm{k}$ and the surface field point.

Define two time-independent variables:

$$
\begin{aligned}
K_{k, j}^{a} & =\frac{\mu_{0}}{4 \pi} \int_{l_{k}} \int_{l_{i}} \frac{1}{\left|r_{k}-r_{i}\right|} d l^{\prime} d l \\
K_{k, j}^{b} & =-\frac{1}{4 \pi \varepsilon} \int_{l_{i}} \frac{1}{\left|r_{k}-r_{i}\right|} d l^{\prime}
\end{aligned}
$$

Equation(13)and(14)can be re-displayed as follows:: 


$$
\begin{gathered}
\Phi_{k, j}=\sum_{i=1}^{N} i_{i, j-n} K_{k, j}^{a} \\
\psi_{i, j}=\sum_{i=1}^{N} i_{i, j-n} K_{k, j}^{b}
\end{gathered}
$$

Substituting equation (17)and (18)into equation (13), The time domain integral equation can be solved.

\section{3 solution of time domain electric field integral equation}

To solve equation (13) the following two aspects need to be considered. First, calculation $\Phi_{k, j}$ and $\psi_{k, j}$ when the current $\mathrm{j}$ moment is unknown, the processing method needs to be considered. Second, due to the existence of time integral. In order to obtain a stable numerical solution, the numerical oscillation of the late response must be considered.

The processing of the first problem is relatively simple, and the equation (13) is arranged to obtain a current at time $\mathrm{j}$ represented by the current at $\mathrm{j}-\mathrm{n}(\mathrm{n}>0)$, thereby solving the problem.

The second problem is the key to the numerical stability, and the current obtained is approximated, $t=t_{j}$, the current on the $k$ segment can be expressed as:

$$
\tilde{I}_{k, j}=\frac{1}{4}\left(\tilde{I}_{k, j-1}+2 I_{k, j}+I_{k, j+1}\right)
$$

After calculating the current at time $j+1$, the current at time $\mathrm{j}$ is re-corrected and the used for subsequent iterative solution, the post-time instability can be eliminated to some extent with using it.

\section{Algorithm verification}

By comparing the induced lightning overvoltage of overhead lines in the same situation, the method is compared with the method provided in [9], verify the calculation method provided in this article

Assume that the length of the overhead line is $1000 \mathrm{~m}$, the diameter of single wire is $1 \mathrm{~cm}$, the height from ground is $10 \mathrm{~m}$, the lightning strike point hits the middle of the line $60 \mathrm{~m}$ from the ground directly above the line, the lighting current propagation speed is $1.3 \times 10^{8} \mathrm{~m} / \mathrm{s}$.the maximum time derivative is $40 \mathrm{kA} / \mu \mathrm{s}$ the peak is $12 \mathrm{kA}$. The lightning overvoltage waveform of the line calculated by simulation is shown in figure 1 :

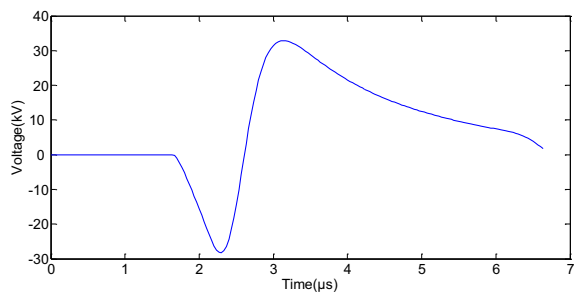

(a) A voltage waveform having an electrical conductivity of $0.001 \mathrm{~m} / \mathrm{s}$

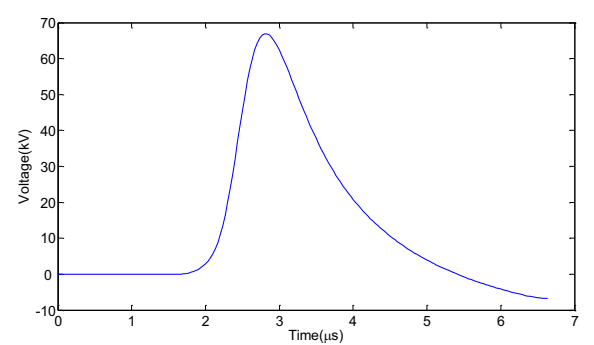

(b) Voltage waveform at infinite conductivity

Fig.1 Lightning induced overvoltage waveforms at different earth electrical conductivity

The calculations show that the difference between the method and the method provided by the literature[10]is very small, which verifies that the method is correct and feasible.

\section{Engineering applications}

The above calculation method can be used to calculate the spatial electromagnetic field generated in the intelligent component cabinet area during the switch operation of the AIS type intelligent substation. taking a $110 \mathrm{kV}$ intelligent substation as a example, two different arrangement of overhead lines are shown in figure 2 .
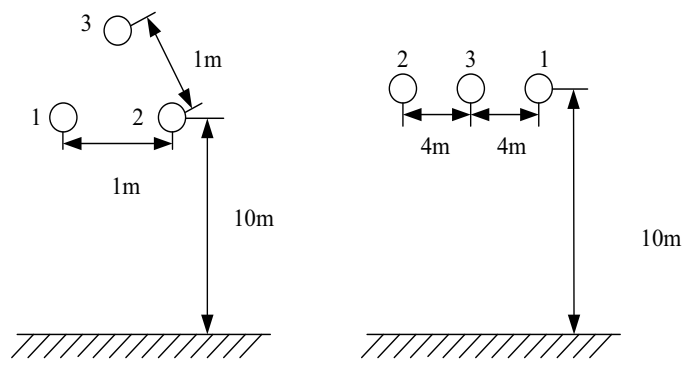

Fig. 2 Two permutations of three conductor overhead lines

Assume that the length of the overhead line is $1000 \mathrm{~m}$, the diameter of single wire is $1 \mathrm{~cm}$, the height from ground is $10 \mathrm{~m}$, the lightning strike point hits the middle of the line $60 \mathrm{~m}$ from the ground directly above the line, the lighting current propagation speed is $1.3 \times 10^{8} \mathrm{~m} / \mathrm{s}$ decaying Changshu is 0.43 . the corresponding matching load is connected at both ends of the line, and the soil is considered to be well conductive. The simulated terminal lightning overvoltage waveform obtained by simulation is shown in figure 3 and 4: 


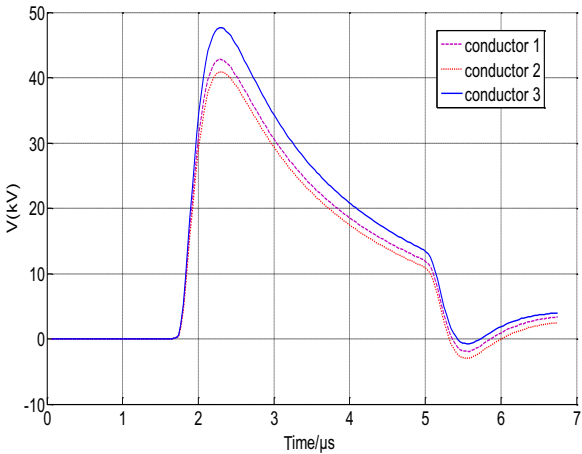

Fig.3 Sensing overvoltage of triangular arrangement overhead line end

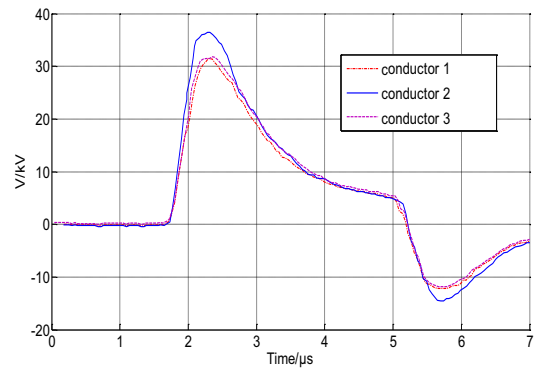

Fig. 4 Sensing overvoltage of Horizontal arrangement overhead line end

\section{Conclusion}

If a certain point in the transmission line is struck by lightning, considering the influence of soil -related physical parameters and long-line horizontal parameters, the derived line-sensing horizontal electric field calculation formula is applicable to any form of lightning. Solving the integral equation to obtain a lightning induced overvoltage by stablishing the time domain equation of the calculation formula.

\section{Acknowledgement}

This work was supported by the Project of SGCC: Research on the influence of VFTO on On-site protection equipment and test technology(JBB17201700082).

\section{References}

1. Wang wenbing. Transient electromagnetic field[M]. Xi'an jiaotong University Press, 1991.

2. Taflove A. Computational electromagnetics: The finite-difference time-domain method[J]. Lmn.pub.ro, 1995.

3. Paul C. Analysis of Multiconductor Transmission Lines[M]// Analysis of multiconductor transmission lines /. Wiley, 1994:45 - 54.

4. Gan zheyuan, Zhao Jun, Zhang Jiangong, et al. Calculation of transient electromagnetic radiation in shell space during operation of GIS isolation switch[J]. High Voltage Technology, 2016, 42(3):980-986.

5. Musa B U, Siew W H, Judd M D. Computation of Transient Electromagnetic Fields Due to Switching in High-Voltage Substations[J]. IEEE Transactions on Power Delivery, 2010, 25(2):1154-1161.

6. Liu L, Cui X, Qi L. Simulation of Electromagnetic Transients of the Bus Bar in Substation by the TimeDomain Finite-Element Method[J]. Electromagnetic Compatibility IEEE Transactions on, 2009, 51(4):1017-1025.

7. P. J. Davies. On the stability of time-marching schemes for the general surface electric-field integral equation[J]. IEEE Trans. Antennas Propagat., 1996, 44:1467-1473

8. Yair Shifman, Yehuda Leviatan. Transient Analysis of EM Pulse Penetration Into a Conducting Layer Using Wavelet-Based Implicit TDIE and Iterative IMC Technique[J]. IEEE Transactions on Antennas and Propagation, 2004, 52(4):1105-1111

9. Farhad Rachidi, Carlo Alberto Nucci, Michel Ianoz, Carlo Mazzetti. Influence of a lossy ground on lightning-induced voltages on overhead lines[J]. IEEE Transactions on Electromagnetic Compatibility, 1996, 38(4): 250-264. 